\title{
DUAL ROLES OF CANCER CELL-EXPRESSED IMMUNOGLOBULINS IN CANCER IMMUNOLOGY
}

\author{
${ }^{1,2}$ Gregory Lee, ${ }^{1}$ Cheng-Yuan Huang, \\ ${ }^{1}$ Suefay Liu, ${ }^{3}$ Chin-Hsiang Chien and ${ }^{4}$ Song-Nan Chow \\ ${ }^{1}$ UBC Centre for Reproductive Health, University of British Columbia, Vancouver, Canada \\ ${ }^{2}$ Department for Pathology, Shantou University Medical College, Shantou, China \\ ${ }^{3}$ Institute of Biochemistry, Yang Ming University, Taipei, Taiwan \\ ${ }^{4}$ Department of Obstretrics and Gynecology, National Taiwan University Hospital, Taipei, Taiwan
}

Received 2014-09-19; Revised 2014-09-23; Accepted 2014-09-30

\begin{abstract}
While the expression of immunoglobulins and $\mathrm{T}$ cell receptors on cancer cells has been well-established for decades, the potential roles and mechanisms of action of these cancerous antigen receptors have not been fully elucidated. A monoclonal antibody designated as RP215, which reacts specifically with the carbohydrate-associated epitope located on the heavy chain region of cancerous immunoglobulins and $\mathrm{T}$ cell receptors, was used as a unique probe to study the roles of antigen receptors in the immunology of cancer cells. Through extensive cell-based biological and immunological studies, it was found that both anti-antigen receptors and RP215 demonstrated similar actions on the gene regulations involved in the growth/proliferation of cancer cells, as well as on toll-like receptors involved in innate immunity. In addition, RP215-specific cancerous immunoglobulins are believed to capture or neutralize circulating antigen/antibodies which may be harmful to cancer cells within the human body. In contrast to normal $\mathrm{B}$ and $\mathrm{T}$ cells and their respective receptors in the conventional immune system, cancer cells coexpress both immunoglobulins and $\mathrm{T}$ cell receptors and immune protection is exercised by unique mechanisms. For example, these cancer cell-expressed antigen receptors display a lack of class switching, limited hyper-mutation, aberrant glycosylations and a strong influence on the toll-like receptors of cancer cells. Therefore, it is hypothesized that both normal and cancerous immune systems may co-exist and operate simultaneously within the human body. The balance of these two immune factors for respective surveillance and protection may be relevant to the outcome of cancer immunotherapy in humans. A potential therapeutic strategy is being developed by using RP215 as a drug candidate to target cancer cells based on these observations.
\end{abstract}

Keywords: Cancerous Immunoglobulins, CA215, RP215 Monoclonal Antibody, Two Immune Systems, Cancer Immunology

\section{INTRODUCTION}

The expressions of antigen receptors, such as immunoglobulins and $\mathrm{T}$ cell receptors, in cancer cells were not discovered until the 1990s (Babbage et al., 2006; Chen and $\mathrm{Gu}, 2007$; $\mathrm{Hu}$ et al., 2011; 2012; Huang et al., 2008; 2009; Kimoto, 1998; Lee and Ge, Corresponding Author: Gregory Lee, UBC Centre for Reproductive

2009; Lee et al., 2008; Li et al., 2001; 2004; Qiu et al., 2003; Zhang et al., 2012; Zheng et al., 2007a; 2007b; 2009; Zhu et al., 2008). In the traditional immune system, it has been well-established that both B and T lymphocytes play differential and interacting roles in our common immune responses against foreign pathogens, such as bacteria and viruses, in the normal human body 
(Murphy et al., 2007). In comparison, the mechanisms of action of antigen receptors widely expressed on the cancer cell surface are not fully understood and require further investigations (Kimoto, 1998).

Based on previous cell-based bioassays with target-specific small interfering Ribonucleic Acid (siRNA) experiments, it was generally concluded that the expressions of cancerous immunoglobulins are essential for the growth/proliferation of cancer cells in vitro and in vivo (Li et al., 2004; 2012; Qiu et al., 2003). However, it remains to be established if the expressions of antigen receptors on the cancer cell surface play any other functional roles in the immunology of cancer cells, including immune protection or evasion (Vince et al., 2007).

A major breakthrough came through in 1987, when a monoclonal antibody designated as RP215 was generated against the cell extract of OC-3-VGH ovarian cancer cell line (Lee et al., 1992). It was later established that RP215 reacts specifically with the carbohydrateassociated epitope located mainly on the heavy chains of cancerous immunoglobulins and cancerous $\mathrm{T}$ cell receptors and not with antigen receptors of the normal immune system (Lee et al., 2006). The group of RP215-specific glycoproteins was termed in general as CA215 and consists mainly of immunoglobulin super family proteins (Lee et al., 2012b). Therefore, RP215 was used as the unique probe for cancerous antigen receptors to study their specific/distinct functional roles in cancer cells. One can then ask if such a cancer immune system has evolved to exist and is being operated independently of our known normal immune system in the human body (Liu et al., 2010).

Since the unexpected discovery of RP215, many biological and immunological studies have been performed during the last decade to investigate the roles of cancerous antigen receptors and elucidate their mechanisms of action in cancer cells (Lee et al., 1992). We believe that the results of these studies may lead to a better strategy in the immunotherapy of cancer cells in humans.

\section{STRUCTURAL COMPARISONS BETWEEN NORMAL AND CANCEROUS IMMUNOGLOBULINS}

By the use of Reverse Transcription Polymerase Chain Reaction (RT-PCR), it was confirmed that the primary amino acid sequences of normal human IgG and cancer cell-expressed $\mathrm{IgG}$ (C-IgG(are virtually identical (Lee et al., 2006). This would indicate that the genes involved in cancerous immunoglobulins and those of normal immunoglobulins are of the same origin in humans (Zheng et al., 2009).

By Western blot assay with OC-3-VGH cancer cell extract and with affinity-purified CA215, RP215 was shown to react with broad protein bands with a molecular size ranging from 50 to $70 \mathrm{KDa}$ (Lee et al., 2008; 2006). Through Matrix-Assisted Laser Desorption/Ionization Time-Of-Flight Mass Spectrometry (MALDI-TOF MS) analysis, the identity of these affinity-purified CA215 was determined to be mainly the heavy chains of immunoglobulins (Lee et al., 2008; 2012b).

A sandwich immunoassay using RP215 as the capturing antibody and horseradish peroxidase (HRP)conjugated RP215 as the detecting antibody was employed to generate dose-dependent signals of CA215 (Fig. 1A). In this assay, it was also demonstrated that goat anti-human IgG-Fc antibodies $\left(1 \mu \mathrm{g} \mathrm{mL} \mathrm{mL}^{-1}\right.$ ) showed little inhibitions on CA215 signals. This is in contrast to goat anti-human IgGFab, where the binding between RP215 and CA215 was greater than $95 \%$ inhibited. Results of such comparative binding assays suggested that the RP215specific "sugar" epitope is localized preferentially in the Fab region of the heavy chain of C-IgG and not in the Fc region (Lee and Azadi, 2012; Lee et al., 2008).

Tunicamycin is known to be a potent inhibitor of $\mathrm{N}$ glycosylation in human cells (Gonzales et al., 2006). No inhibition of RP215 binding to cancer cells was observed in the presence of high doses of tunicamycin within the cancer cell culture (Fig. 1B). This observation would indicate that the carbohydrate-associated epitope recognized by RP215 is not N-glycan-linked, but Olinked. Extensive glycoanalysis was performed with affinity-purified CA215, including CA215-D, which was obtained through a further purification with goat anti-human IgG affinity column. The preliminary results of O-linked glycans associated with CA215 are presented in Table 1 (Lee and Azadi, 2012). Similar O-linked glycan structures were detected when those of CA215 obtained from OC-3-VGH ovarian and C33A cervical cancer cell lines was compared, respectively. From this glycoanalysis, several Olinked oligoglycans were consistently detected. The details of the elucidated structural linkage relationships are still being worked out (Lee and Azadi, 2012). 


\section{CLASS SWITCHING AND SOMATIC HYPERMUTATION OF NORMAL AND CANCEROUS IMMUNOGLOBULINS}

Normal immunoglobulins are expressed through specific class switching processes and serial somatic hypermutations (Honjo et al., 2002; Muramatsu et al., 2000; Papavasiliou and Schatz, 2002). In contrast, cancerous immunoglobulins can be produced from a single clone with many classes and subclasses expressed simultaneously (Early et al., 1980; Honjo et al., 2002). Based on the molecular cloning analysis, a limited diversity of immunoglobulins with restricted somatic mutations $(\leq 20 \%)$ was found to be expressed in cancer cells (Early et al., 1980; Honjo et al., 2002). The octamer element-2 (5'-ATGCAAT-3') (Oct-2) located in the immunoglobulin promoter region was determined to be crucial to cancerous IgG expression (Zhu et al., 2010).

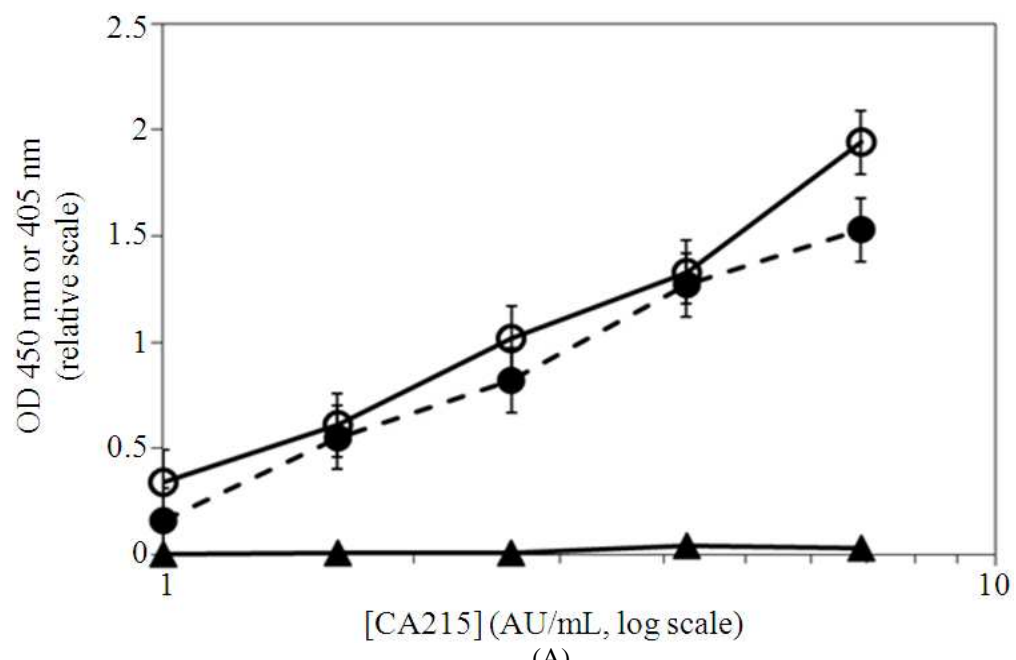

(A)

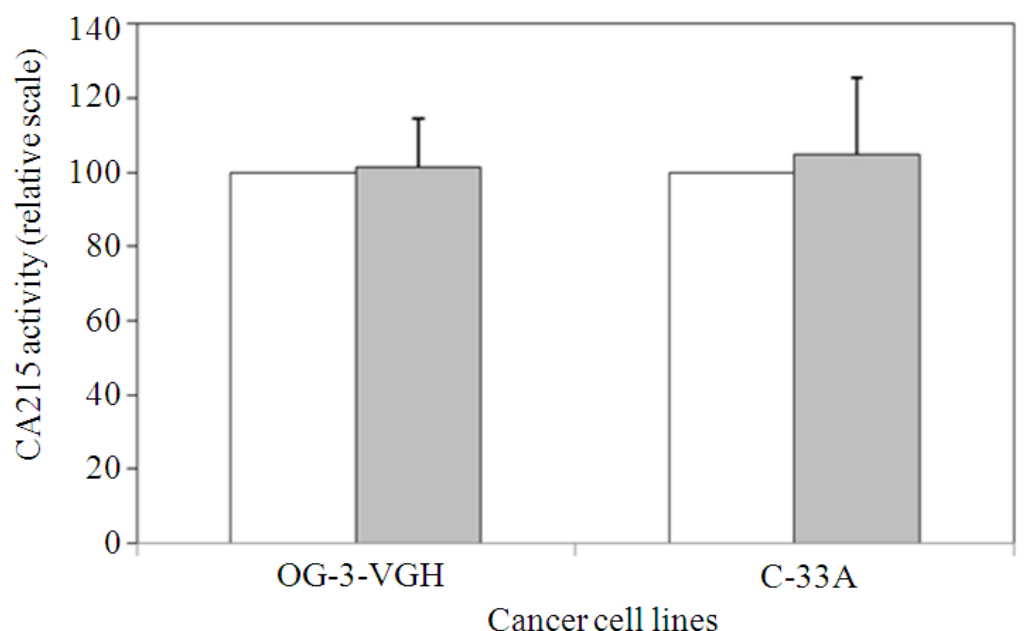

(B)

Fig. 1. Activity of CA215. (A): RP215-based enzyme immunoassay to reveal dose-dependent signal of CA215 (०) (expressed in $\mathrm{AU} / \mathrm{ml})$ and ef-fects of goat anti-human $\operatorname{IgG}-\mathrm{Fc}(\bullet)\left(1 \mu \mathrm{gL}^{-1}\right)$ and anti-human IgG-Fab $(\boldsymbol{\Lambda})\left(1 \mu \mathrm{g} \mathrm{mL}^{-1}\right)$ on CA215 signal intensity. Details of a typical RP215 enzyme immunoassay are described previously (Lee and Ge, 2009; Lee et al., 2008). (B): Effects of $48 \mathrm{hr}$ tunicamycin treatments $\left(1 \mu \mathrm{g} \mathrm{mL} \mathrm{m}^{-1}\right)$ on CA215 activity in the supernatant of two cultured cancer cells for OC-3-VGH and C-33A. To each cell line the left column is the negative control (assuming 100\% activity) without drugtreatment and the right column is the relative CA215 activity following the drug treatment (expressed in triplicate) 
Table 1. Comparative profiles of permethylated O-linked glycans of five different CA215 samples which consist mainly of cancerous $\mathrm{IgG}$

\begin{tabular}{|c|c|c|c|}
\hline Sample ID & Observed Mass m/z [M+Na]+ & Proposed structure & Structure \\
\hline CA215 (lots: A, B and C) ${ }^{\mathrm{a}}$ & 534 & GalNAc1Gal1 & \\
\hline CA215 (lots: A and B) & 708 & GalNAc1Gal1Fuc1 & \\
\hline CA215 (lots: A, B, D, C, E and F) & 896 & GalNAc1Gal1NeuAc1 & $\diamond-a-a$ or \\
\hline CA215 (lots: C, E and F) & 926 & GalNAc1Gal1NeuGc1 & \\
\hline CA215C & $940^{\mathrm{c}}$ & GalNAc1GlcNAc1NeuAc1 & \\
\hline CA215 (lots: A, B and C) & 1140 & GalNAc1GlcNAc1Gal1NeuAc1 & \\
\hline CA215 (lots: C, D, E and F) ${ }^{\mathrm{b}}$ & 1257 & GalNAc1Gal1NeuAc2 & \\
\hline CA215 (lots: C, E and F) & 1317 & GalNAc1Gal1NeuGc2 & $\diamond-\sigma$ \\
\hline CA215 (lots: A, B, C, E and F) & 1345 & GalNAc1GlcNAc1Gal2NeuAc1 & \\
\hline CA215 (lots: C, E and F) & 1375 & GalNAc1GlcNAc1Gal2NeuGc1 & \\
\hline
\end{tabular}

${ }^{\mathrm{a}} \mathrm{CA} 215$ lots A, B and C were from OC-3-VGH ovarian cancer cells lots A and B were obtained through acid elution, whereas lots C, D, E and $\mathrm{F}$ were obtained through elution with $3 \mathrm{M}$ urea. ${ }^{\mathrm{b}}$ Lot $\mathrm{CA} 215 \mathrm{D}$ was obtained by an additional purification of urea-eluted CA215 with goat anti-human IgG affinity column followed by the same analysis (CA215D is designated as affinity-purified cancerous IgG). CA215 lots E and $\mathrm{F}$ were from C-33A cervical cancer cells. ${ }^{c}$ Detected by MALDI-TOF MS method but not found by NSI-MS method. ${ }^{\mathrm{N}} \mathrm{N}-$ acetylgalactosamine $(\square)$, N-acetylglucosamine $(\bullet)$, Fucose $(\boldsymbol{\Delta})$, Galactose $(\bullet)$, N-acetylneuraminic acid $(\bullet)$ and N-glycolylneuraminic acid $(\diamond)$. Obtained from (Lee and Azadi, 2012) with permission

In contrast, octamer element-1 (Oct-1) is essential in normal B cells and is used as transcriptional factor (Zheng et al., 2009). These observations show that the class switching phenomenon, which occurs in normal B cells, does not take place in immunoglobulins expressed by cancer cells. There is also little diversity of immunoglobulins expressed by cancer cells due to limited somatic hypermutations in the cancerous immune system (Early et al., 1980; Honjo et al., 2002; Zheng et al., 2009).

The molecular nature of cancerous immunoglobulin genes has been previously explored (Zheng et al., 2009). For example, seven dominant specific restricted patterns of $\mathrm{V}_{\mathrm{H} \gamma} \mathrm{DJ}_{\mathrm{H} \gamma}$ recombination have been found in cancerous immunoglobulins. However, the mechanisms of hypermutation were different from antigen selection in the B-cell-derived $\mathrm{V}_{\mathrm{H} \gamma} \mathrm{DJ}_{\mathrm{H} \gamma}$ sequence. Therefore, a distinct repertoire of $\mathrm{V}_{\mathrm{H} \gamma} \mathrm{DJ}_{\mathrm{H} \gamma}$ must exist for cancerous immunoglobulins.

\section{GENE REGULATION STUDIES OF CANCEROUS IMMUNOGLOBULINS}

Attempts were made to study the changes in gene expression of cultured cancer cells in response to challenge by RP215, anti-human IgG, or anti-T-cell receptors
(Zhou et al., 2006). Among these were genes involved in the regulation of the cell cycle (cyclin D1, P21, c-fos), protein synthesis (ribosomal proteins $\mathrm{P}_{0}, \mathrm{P}_{1}$ ), regulation of growth (Epidermal Growth Factor Receptor (EGFR), IgG, $\mathrm{T}$ Cell Receptor (TCR), Nuclear Factor kappa-B p105 subunit 1 (NFאB-1)) and Toll-Like Receptors (TLR-2, 3, 4, 9 ), which are essential for the innate immunity of cancer cells (Tang et al., 2013). Based on the results of these extensive studies with semi-quantitative RT-PCR, efforts were made to analyze if the expressions or regulations of these genes are correlated by the presence of any of these three anti-antigen receptor ligands (Zhou et al., 2006).

The results of such comparative gene expression analysis are presented in Table 2 (Vince et al., 2007; Zhou et al., 2006). A high degree of consistency in the changes on gene regulation by these three anti-antigen receptors was observed. The correlation coefficients range from $0.90-0.95$ when the pair-wise statistical analyses are performed with either OC-3-VGH ovarian or C-33A cervical cancer cell line as the testing model. Among these genes, those of toll-like receptors were strongly affected by RP215 or any other of the two antiantigen receptors (Zhou et al., 2006). The results of these gene regulation studies seem to suggest that NFKB-1 gene is highly involved in the regulation of these genes involved in the growth/proliferation of cancer cells and 
therefore is crucial to the immunology of cancer cells (Ditsworth and Zong, 2004; Gellman, 1997; Rokach et al., 1980; Xie et al., 2009; Young, 2012).

\section{DETECTION OF BINDING SUBSTANCES OF CA215 OR CANCEROUS IMMUNOGLOBULINS IN POOLED HUMAN SERUM SPECIMENS}

Attempts were made to identify any binding proteins/components exhibiting affinity to CA215 or C-
IgG. Purified CA215 and/or cancerous immunoglobulins were used as the ligand to capture binding substances or proteins in human circulation (Lee et al., 2014a). The affinity-purified proteins isolated from pooled human serum (CA215 S+) were coated on microwells (Fig. 2). Biotinylated CA215 or cancerous IgG were shown to exhibit dose-dependent binding relationship to CA215 and $\mathrm{C}-\mathrm{IgG}$ by Enzyme Immunoassay (EIA). The presence of CA215 or C-IgG-associated binding proteins or substances in pooled human serum specimens was clearly confirmed by binding ELISA in this study.

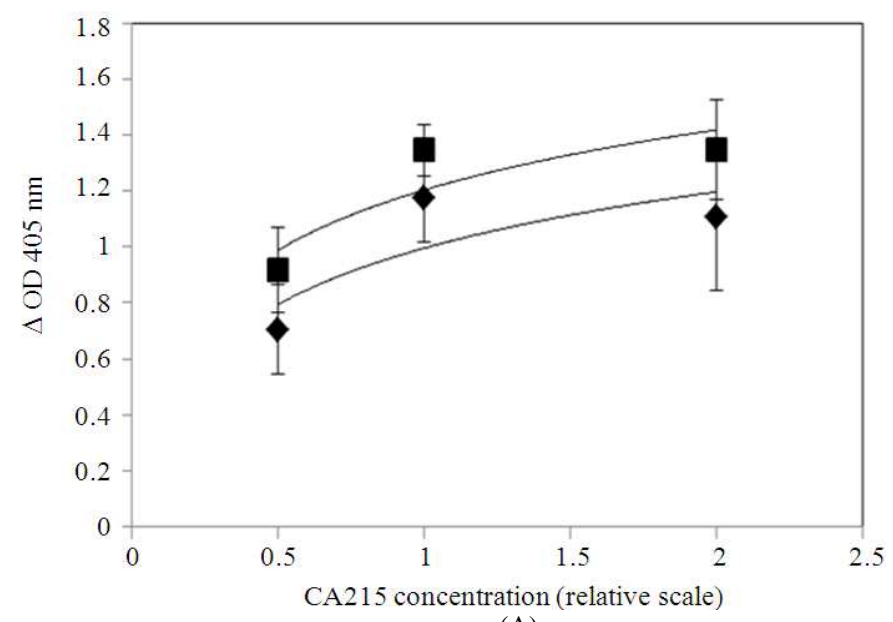

(A)

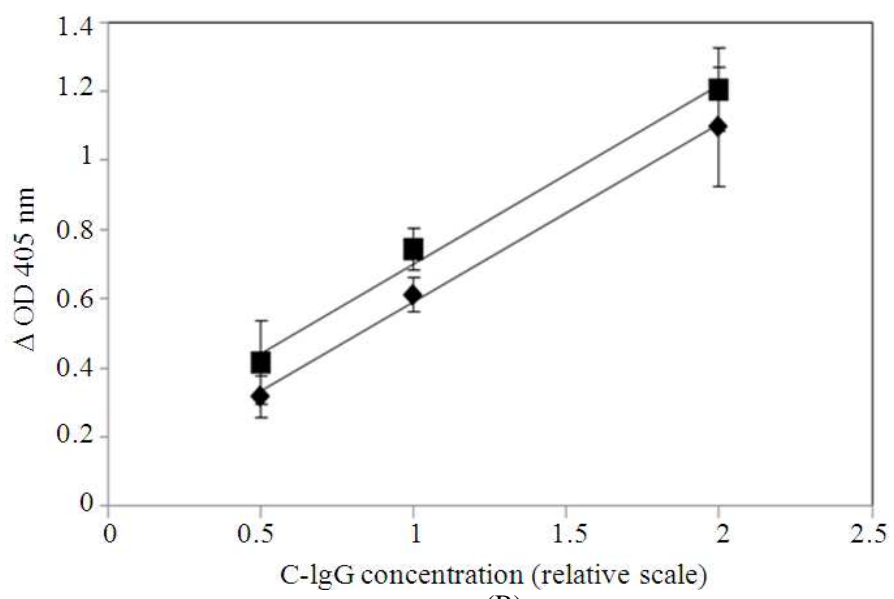

(B)

Fig. 2. Dose-dependent EIA binding activity between CA215 or C-IgG and microwells coated with affinity-purified binding proteins isolated from pooled human serum specimens. Affinity purified CA215 or C-IgG were first biotinylated prior to EIA binding studies. (A): Biotinylated CA215 versus C-IgG S+ coated wells (๘), or CA215 S+ coated wells $(\star)$. The signal intensity was determined with $3 \mathrm{hr}$ binding incubation at $37^{\circ} \mathrm{C}$, then for signal detection, alkaline-phosphatase-labeled avidin was used for additional one hour incubation at $37^{\circ} \mathrm{C}$, followed by adding substrate for signal detection. Details were described previously (Lee et al., 2014a). (B): Biotinylated C-IgG versus C-IgG S+ coated wells (๘), or CA215 S+ coated wells ( $\downarrow$ ). Experimental conditions are the same as those in $(\mathrm{A})$ 
Table 2. Effects of treatments of cultured OC-3-VGH ovarian or C-33A cervical cancer cells with Goat anti-human IgG (GahIgG), rabbit anti-T Cell Receptors $\beta(\operatorname{RaTCR} \beta)$ and murine RP215 (mRP215) on the expressions of genes involved in cell prolife-ration, protein synthesis, cell cycle regulations and the innate immunitya

\begin{tabular}{|c|c|c|c|c|c|c|}
\hline \multirow[b]{2}{*}{ Gene } & \multicolumn{2}{|l|}{ GahIgG } & \multicolumn{2}{|l|}{$\mathrm{R} \alpha \mathrm{TCR} \beta$} & \multicolumn{2}{|l|}{ mRP215 } \\
\hline & OC-3-VGH & $\mathrm{C}-33 \mathrm{~A}$ & OC-3-VGH & $\mathrm{C}-33 \mathrm{~A}$ & OC-3-VGH & $\mathrm{C}-33 \mathrm{~A}$ \\
\hline IgG & 0 & $\Delta \mathbf{\Delta \Delta}$ & 0 & $\Delta \mathbf{\Delta} \mathbf{\Delta}$ & 0 & $\Delta \mathbf{\Delta} \Delta$ \\
\hline $\mathrm{T}$ cell receptor & $\triangle$ & 0 & $\triangle \triangle \triangle$ & $\Delta$ & $\triangle \triangle \triangle$ & 0 \\
\hline NFKB-1 & $\triangle \triangle \triangle$ & $\mathbf{\Delta} \mathbf{\Delta} \mathbf{\Delta}$ & $\triangle \triangle \triangle$ & $\mathbf{\Delta} \mathbf{\Delta} \mathbf{\Delta}$ & $\triangle \triangle \triangle$ & $\Delta \mathbf{\Delta} \mathbf{\Delta}$ \\
\hline Cyclin D1 & $\nabla$ & $\nabla \nabla$ & $\nabla \nabla$ & $\nabla$ & $\nabla \nabla \nabla$ & $\mathbf{\Delta} \mathbf{\Delta} \mathbf{A}$ \\
\hline $\mathrm{P} 21$ & $\triangle \triangle \triangle$ & $\boldsymbol{\Delta}$ & $\triangle \triangle \triangle$ & 0 & $\triangle \triangle \triangle$ & $\Delta$ \\
\hline c-fos & $\nabla$ & $\mathbf{\Delta} \mathbf{\Delta} \boldsymbol{\Delta}$ & 0 & 0 & $\nabla$ & $\nabla \nabla$ \\
\hline $\mathrm{P}_{\mathrm{o}}$ & $\triangle \triangle \triangle$ & NA & $\nabla \nabla \nabla$ & NA & 0 & NA \\
\hline $\mathrm{P}_{1}$ & $\triangle \triangle$ & $\Delta$ & $\triangle$ & $\Delta \mathbf{\Delta}$ & $\triangle$ & $\Delta \mathbf{\Delta} \mathbf{\Delta}$ \\
\hline EGFR & $\triangle \triangle \triangle$ & NA & $\nabla \nabla \nabla$ & NA & $\nabla \nabla \nabla$ & NA \\
\hline TLR-2 & NA & $\Delta \boldsymbol{\Delta}$ & NA & $\Delta$ & NA & $\mathbf{\Delta} \mathbf{\Delta} \boldsymbol{\Delta}$ \\
\hline TLR-3 & $\triangle \triangle \triangle$ & $\mathbf{\Delta} \mathbf{\Delta} \boldsymbol{\Delta}$ & $\triangle \triangle \triangle$ & $\mathbf{\Delta} \mathbf{\Delta} \boldsymbol{\Delta}$ & $\triangle \triangle \triangle$ & $\Delta \mathbf{\Delta} \mathbf{\Delta}$ \\
\hline TLR-4 & $\nabla \nabla \nabla$ & $\nabla \nabla \nabla$ & $\nabla \nabla \nabla$ & $\nabla$ & $\nabla \nabla \nabla$ & \\
\hline TLR-9 & $\nabla \nabla \nabla$ & $\nabla \nabla \nabla$ & $\nabla \nabla \nabla$ & $\nabla \nabla$ & $\nabla \nabla \nabla$ & $\nabla \nabla \nabla$ \\
\hline
\end{tabular}

${ }^{\mathrm{a}}(0)$ less than $10 \%$ gene expression increase or decrease, ( $\triangle$ or $\left.\boldsymbol{\Delta}\right)$ 10-20\% gene expression increase, $(\triangle \triangle$ or $\boldsymbol{\Delta} \mathbf{\Delta})$ 20-30\% gene expression increase, $(\triangle \triangle \triangle$ or $\mathbf{\Delta} \mathbf{\Delta} \mathbf{\Delta})$ above $30 \%$ gene expression increase, $(\nabla$ or $\boldsymbol{\nabla}) 10-20 \%$ gene expression decrease, $(\nabla \nabla$ or $\boldsymbol{\nabla \nabla})$ 20-30\% gene expression decrease, $(\nabla \nabla \nabla$ or $\boldsymbol{\nabla \nabla \nabla}$ ) above $30 \%$ gene expression decrease, (NA) not available. Modified from (Tang et al., 2013) with permission

These binding proteins could be of antibody or antigen origins or of other origins (Lee et al., 2014a). Based on these observations, it seems reasonable to assume that cancerous immunoglobulins or CA215 displayed on the cancer cell surface may have functional activities to capture or neutralize unidentified substances in pooled human serum specimens as a means of immune protection for the cancer cells (Lee et al., 2014a). Conversely, these CA215 or C-IgG-specific substances might be present in normal human circulation to neutralize or inactivate cancer cells. The reasons for the presence of these binding substances remain to be investigated. It is currently unknown whether these substances are naturally occurring or induced through appropriate immune responses to circulating cancer cells in the human immune system.

\section{BIOLOGICAL AND IMMUNOLOGICAL ACTIVITITES OF RP215 OR OTHER ANTI-ANTIGEN RECEPTORS ON CANCER CELLS}

Since C-IgGs are absolutely required for the growth/proliferation or survival of cancer cells in vitro or in vivo, RP215 or anti-antigen receptors were used as a probe in induced apoptosis studies in cultured cancer cells to demonstrate RP215's anti-proliferative actions (Chen and Gu, 2007; Lee et al., 2012a; Li et al., 2012;
Qiu et al., 2003; Zheng et al., 2007a). Results of such studies are presented in Fig. 3A. Through these studies, it was demonstrated that apoptosis can be induced in cultured cancer cells by a number of anti-antigen receptors, including RP215, anti-IgG, anti-IgM, anti-IgA, anti-Ig $\lambda$ and anti-TCR- $\beta$. Interestingly, anti-TLR-4 was also found to induce apoptosis in cultured cancer cells following the same incubation conditions. Similarly, RP215 was also shown to induce Complement-Dependent Cytotoxicity (CDC) reactions (Lee et al., 2012a). CDC reactions can also be induced in cancer cells upon incubation with many anti-antigen receptors. The results are shown in Fig. 3B for comparative purposes.

The results of these two cell-based biological assays clearly demonstrate that RP215 has anti-cancer properties and its humanized forms demonstrate the potential to be developed as anti-cancer drugs in humans for future therapeutic applications.

\section{HYPOTHESIS OF TWO INDEPENDENT IMMUNE SYSTEMS AND THE DUAL ROLES OF CANCEROUS IMMUNOGLOBULINS}

In this review, experimental observations have been summarized and presented to indicate that two independent immune systems may exist in the human body (Lee et al., 2014a). 

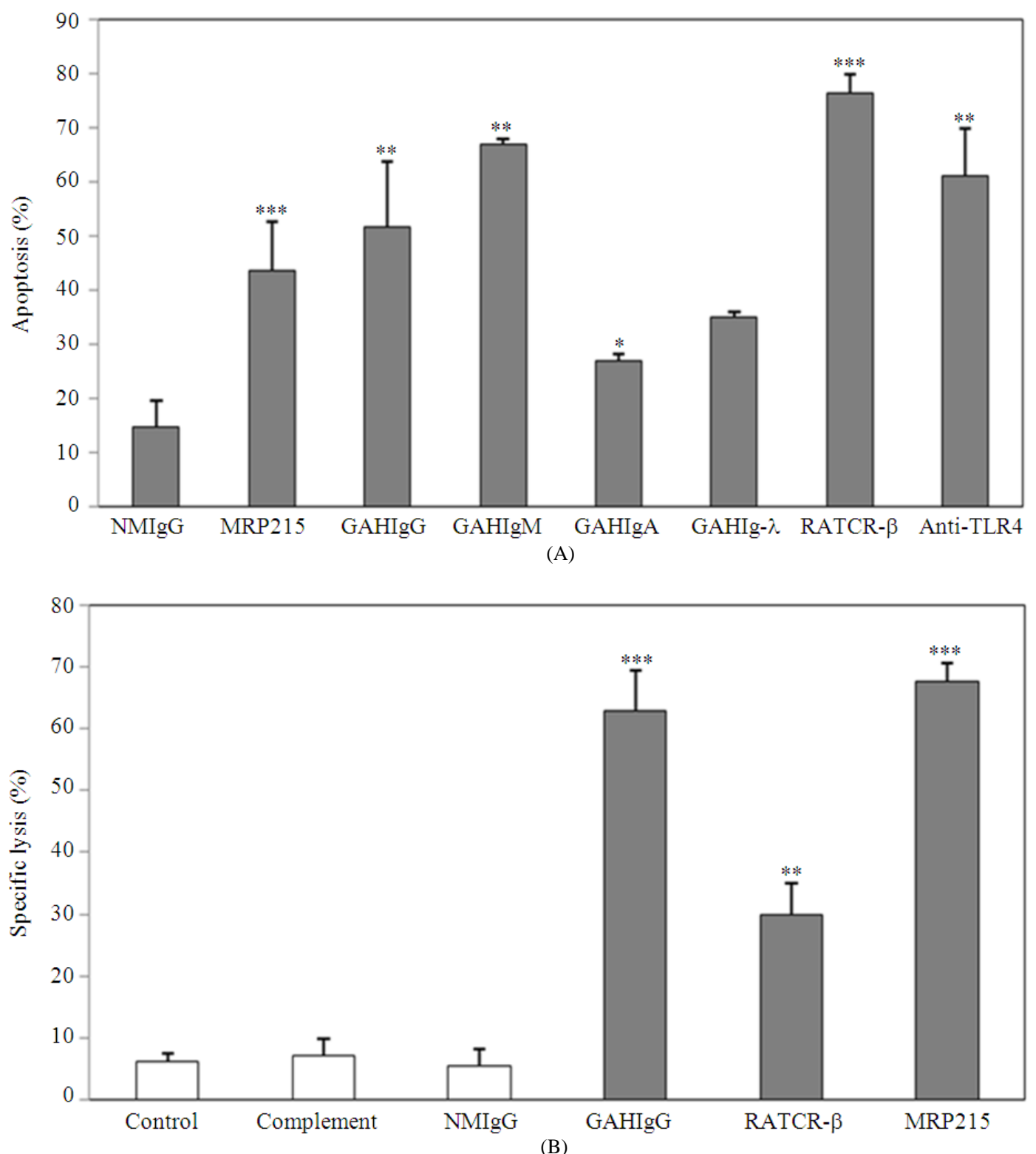

Fig. 3. Induced apoptosis of OC-3-VGH cancer cells. (A): Induced apoptosis on treated cultured OC-3-VGH cancer cells by Normal Mouse IgG (NMIgG) (Lane 1), Murine RP215 (MRP215) (Lane 2), (Lane 3), Goat Anti-Human IgM (GAHIgM) (Lane 4), Goat Anti-Human IgA (GAHIgA) (Lane 5), Anti-Human $\lambda$ light Goat Anti-Human IgG (GAHIgG) chain (GAHIg $\lambda$ ) (Lane 6), Rabbit anti-T cell receptor $\beta$ (RATCR- $\beta$ ) (Lane 7) and anti-TLR4 (Lane 8) for $24 \mathrm{~h}$ incubation. $10 \mu \mathrm{g} \mathrm{mL}{ }^{-1}$ of the ligands were added to cancer cell culture medium for the apoptosis assay, by using terminal deoxynucleotidyl Transferase dUTP Nick End Labeling (TUNEL) assay kit from Roche Diagnostics (Lee et al., 2012a). (B): The Complement Dependent Cytotoxicity (CDC) assay to demonstrate the effect of goat anti-human IgG, anti-T cell receptor $\beta$ and RP215 on complement-dependent cytotoxicty of OC-3-VGH ovarian cancer cell line; Lane 1: no treatment (control), Lane 2: complement only, Lane 3: Normal Mouse IgG and complement (NMIgG), Lane 4: Goat Anti-Human IgG and complement (GAHIgG), Lane 5: Rabbit Anti-T Cell Receptor $\beta$ and complement (RATCR- $\beta$ ), Lane 6: Murine RP215 and complement (MRP215). *,**,*** indicate statistical significance of $\mathrm{p}<0.05, \mathrm{p}<0.01$ and $\mathrm{p}<0.001$, respectively. Both (A) and (B) were modified from (Lee et al., 2012a) with permission 
One is the common normal immune system, in which B and $\mathrm{T}$ lymphocytes play major roles in generating immunoglobulins and $\mathrm{T}$ cell receptors of high diversity through a series of interactions, differentiations and productions of effector molecules, including antibodies. An unlimited number of antibodies can be produced and released into circulation to fight off foreign pathogens such as viruses, fungi and bacteria (Lee et al., 2014b, Murphy et al., 2007; Lee et al., 2014a). Our recent studies show that CA215 or CIgG-specific binding proteins can be detected in pooled human serum specimens and react with cancerous immunoglobulins (Lee et al., 2014a). It remains to be demonstrated if these binding proteins or substances are antigens, antibodies, or other molecules which may be induced or occurring naturally (Lee et al., 2014a).

Cancer cells might have their own immune system which can be distinguished from the conventional immune system within the human body. As mentioned previously, normal immunoglobulins and their cancerous counterparts can be differentiated by comparison of their mutual glycosylation patterns, class switching mechanisms, hypermutation, influences on innate immunity and apparent immunoactivity. Therefore, it is reasonable to assume that a separate independent immune system should be available for the growth/proliferation and immune protections of cancer cells under our normal human body environment. In fact, the existence of two separate immune systems is quite efficient and logical from an evolutionary point of view. Since cancer cells are recognized as "foreign" in our human body, the cancer immune system is one of the most effective means for immune protection and growth/proliferation of cancer cells. Any strategy resulting in the deactivation of this cancer immune system should lead to effective immunotherapy of relevant cancer cells in humans. Therefore, the widespread expressions of these cancerous immunoglobulins can be a potential target for cancer immunotherapy.

\section{ACKNOLWEDGMENTS}

This research project was supported in parts by NRCIRAP program (\#794354) of Canada. Helpful discussion on this topic with Dr. Bixia Ge is acknowledged.

\section{REFERENCES}

Babbage, G., C. Ottensmeier, J. Blaydes, F. Stevenson and S. Sahota, 2006. Immunoglobulin heavy chain locus events and expression of activation-induced cytidine deaminase in epithelial breast cancer cell lines. Cancer Res., 66: 3996-4000. DOI: 10.1158/0008-5472.CAN-05-3704
Chen, Z. and J. Gu, 2007. Immunoglobulin g expression in carcinomas and cancer cell lines. FASEB J., 21: 2931-2938. DOI: 10.1096/fj.07-8073

Ditsworth, D. and W.X. Zong, 2004. Nf-kappab: Key mediator of inflammation-associated cancer. Cancer Biol. Therapy, 3: 1214-1216. PMID: 15611628.

Early, P., H. Huang, M. Davis, K. Calame and L. Hood, 1980. An immunoglobulin heavy chain variable region gene is generated from three segments of DNA: Vh, d and jh. Cell Divis. Biol. California Institute Technol. Pasadena, 19: 981992. DOI: 10.1016/0092-8674(80)90089-6

Gellman, S.H., 1997. Introduction: Molecular recognition. Chem. Rev., 97: 1231-1232. DOI: $10.1021 / \mathrm{cr} 970328 \mathrm{j}$

Gonzales, D., S.I. Rennard, M. Nides, C. Oncken and K.R. Reeves et al., 2006. Varenicline, an $\alpha 4 \beta 2$ nicotinic acetylcholine receptor partial agonist, vs sustained-release bupropion and placebo for smoking cessation: A randomized controlled trial. J. Am. Med. Associat., 296: 47-55. DOI: 10.1001/jama.296.1.47

Honjo, T., K. Kinoshita and M. Muramatsu, 2002. Molecular mechanism of class switch recombination: Linkage with somatic hypermutation. Ann. Rev. Immunol., 20: 165-196. DOI: 0.1146/annurev.immunol.20.090501.112049

Hu, D., Z. Duan, M. Li, Y. Jiang and H. Liu et al., 2011. Heterogeneity of aberrant immunoglobulin expression in cancer cells. Cellular Molecular Immunol., 8: 479-485. DOI: 10.1038/cmi.2011.25

$\mathrm{Hu}, \mathrm{F}$., L. Zhang, J. Zheng, L. Zhao and J. Huang et al., 2012. Spontaneous production of immunoglobulin $\mathrm{m}$ in human epithelial cancer cells. PLoS One, 7: e51423- e51423. DOI: 10.1371/journal.pone.0051423

Huang, J., X. Sun, Y. Mao, X. Zhu and P. Zhang et al., 2008. Expression of immunoglobulin gene with classical $\mathrm{v}-(\mathrm{d})-\mathrm{j}$ rearrangement in mouse brain neurons. Int. J. Biochem. Cell Biol., 40: 16041615. DOI: $10.1016 /$ j.biocel.2007.12.004

Huang, J., L. Zhang, T. Ma, P. Zhang and X. Qiu, 2009. Expression of immunoglobulin gene with classical $\mathrm{v}-(\mathrm{d})-\mathrm{j}$ rearrangement in mouse testis and epididymis. J. Histochem. Cytochem., 57: 339349. DOI: $10.1369 /$ jhc.2008.951434 
Kimoto, Y., 1998. Expression of heavy-chain constant region of immunoglobulin and t-cell receptor gene transcripts in human non-hematopoietic tumor cell lines. Genes Chromosomes Cancer, 22: 8386. DOI: $10.1002 /$ (sici) 1098 2264(1998)22:1<83::aid-gcc12>3.0.co;2-o

Lee, C.Y., K.W. Chen, F.S. Sheu, A. Tsang and K.C. Chao et al., 1992. Studies of a tumor-associated antigen, cox-1, recognized by a monoclonal antibody. Cancer Immunol. Immunotherapy, 35: 19-26. DOI: 10.1007/BF01741050

Lee, G. and B. Ge, 2009. Cancer cell expressions of immunoglobulin heavy chains with unique carbohydrate-associated biomarker. Cancer Biomarkers, 5: 177-188. DOI: 10.3233/cbm-20090102

Lee, G. and P. Azadi, 2012. Peptide mapping and glycoanalysis of cancer cell-expressed glycoproteins ca215 recognized by $\mathrm{rp} 215$ monoclonal antibody. J. Carbohydrate Chem., 31: 10-30. DOI: 10.1080/07328303.2011.626544

Lee, G., A. Cheung, B. Ge, M. Zhu and B. Giolma et al., 2012a. Ca215 and gnrh receptor as targets for cancer therapy. Cancer Immunol. Immunotherapy, 61: 1805-1817. DOI: 10.1007/s00262-012-1230-8

Lee, G., M. Zhu, B. Ge and S. Potzold, 2012b. Widespread expressions of immunoglobulin superfamily proteins in cancer cells. Cancer Immunol., Immunotherapy, 61: 89-99. DOI: 10.1007/s00262-011-1088-1

Lee, G., C.Y. Huang, Y. Tang and H. Zhang, 2014a. Potential roles of cancerous immunoglobulins in the immunology of cancer cells. J. Clin. Cellular Immunol., 5: 1-7. DOI: 10.4172/21559899.1000200

Lee, G., C.Y. Huang, H. Zhang and Y. Tang, 2014b. The relationships between toll-like receptors and rp215-associated immunoglobulins expressed by cancer cells. J. Cancer Sci. Therapy, 6: 77-80. DOI: $10.4172 / 1948-5956.1000252$

Lee, G., E. Laflamme, C.H. Chien and H.H. Ting, 2008. Molecular identity of a pan cancer marker, ca215. Cancer Biol. Therapy, 7: 2007-2014. DOI: 10.4161/cbt.7.12.6984

Lee, G., Q. Wu, C.H. Li, H.H. Ting and C.H. Chien, 2006. Recent studies of a new carbohydrateassociated pan cancer marker, ca215. J. Clin. Ligand Assay, 29: 47-51.
Li, J., C. Tan, Q. Xiang, X. Zhang and J. Ma et al., 2001. Proteomic detection of changes in protein synthesis induced by ngx6 transfected in human nasopharyngeal carcinoma cells. J. Prot. Chem., 20: 265-271. DOI: 10.1023/a:1010912311564

Li, M., D.Y. Feng, W. Ren, L. Zheng and H. Zheng et al., 2004. Expression of immunoglobulin kappa light chain constant region in abnormal human cervical epithelial cells. Int. J. Biochem. Cell Biol., 36: 2250-2257. DOI: 10.1016/j.biocel.2004.03.017

Li, M., H. Zheng, Z. Duan, H. Liu and D. Hu et al., 2012. Promotion of cell proliferation and inhibition of adcc by cancerous immunoglobulin expressed in cancer cell lines. Cellular Molecular Immunol., 9: 54-61. DOI: $10.1038 / \mathrm{cmi} .2011 .40$

Liu, J.Z., F. Tozzi, D.M. Waterworth, S.G. Pillai and P. Muglia et al., 2010. Meta-analysis and imputation refines the association of $15 \mathrm{q} 25$ with smoking quantity. Nature Genet., 42: 436-440. DOI: 10.1038/ng.572

Muramatsu, M., K. Kinoshita, S. Fagarasan, S. Yamada and Y. Shinkai et al., 2000. Class switch recombination and hypermutation require activation-induced cytidine deaminase (aid), a potential rna editing enzyme. Cell, 102: 553-563. DOI: 10.1016/s0092-8674(00)00078-7

Murphy, K., P. Travers and M. Walport, 2007. Janeway's immunobiology. 1st Edn., Garland Science, New York, ISBN-10: 9780815341239, pp: 323-496.

Papavasiliou, F.N. and D.G. Schatz, 2002. Somatic hypermutation of immunoglobulin genes: Merging mechanisms for genetic diversity. Cell, 109: 35-44.

Qiu, X., X. Zhu, L. Zhang, Y. Mao and J. Zhang et al., 2003. Human epithelial cancers secrete immunoglobulin $\mathrm{g}$ with unidentified specificity to promote growth and survival of tumor cells. Cancer Res., 63: 6488-6495.

Rokach, J., Y. Girard, Y. Guindon, J.G. Atkinson and M. Larue et al., 1980. The synthesis of a leukotriene with srs-like activity. Tetrahedron Lett., 21: 1485-1488. DOI: 10.1016/S00404039(00)92753-9

Tang, Y., H. Zhang and G. Lee, 2013. Similar gene regulation patterns for growth inhibition of cancer cells by rp215 or anti-antigen receptors. J. Cancer Sci. Therapy, 5: 200-208. DOI: 10.4172/19485956.1000207 
Vince, J.E., W.W.L. Wong, N. Khan, R. Feltham and D. Chau et al., 2007. Iap antagonists target ciap1 to induce tnf $\alpha$-dependent apoptosis. Cell, 131: 682693. DOI: 10.1016/j.cell.2007.10.037

Xie, W., Y. Wang, Y. Huang, H. Yang and J. Wang et al., 2009. Toll-like receptor 2 mediates invasion via activating nf-kappab in mda-mb-231 breast cancer cells. Biochem. Biophysical Res. Commun., 379: 1027-1032. DOI: 10.1016/j.bbrc.2009.01.009

Young, R.N., 2012. Discovery and Development of Montelukast (singulairß). In: Case Studies in Modern Drug Discovery and Development, Huang, X. and R.G. Aslanian (Eds.)., John Wiley and Sons, Hoboken, ISBN-10: 1118219708 , pp: 528.

Zhang, L., S. Hu, C. Korteweg, Z. Chen and Y. Qiu et al., 2012. Expression of immunoglobulin $\mathrm{g}$ in esophageal squamous cell carcinomas and its association with tumor grade and ki67. Human Pathol., 43: 423-434. DOI: 10.1016/j.humpath.2011.05.020

Zheng, H., M. Li, H. Liu, W. Ren and D.S. Hu et al., 2007a. Immunoglobulin alpha heavy chain derived from human epithelial cancer cells promotes the access of $\mathrm{s}$ phase and growth of cancer cells. Cell Biol. Int., 31: 82-87. DOI: 10.1016/j.cellbi.2006.09.009
Zheng, H., M. Li, W. Ren, L. Zeng and H.D. Liu et al., 2007b. Expression and secretion of immunoglobulin alpha heavy chain with diverse vdj recombinations by human epithelial cancer cells. Molecular Immunol., 44: 2221-2227. DOI: 10.1016/j.molimm.2006.11.010

Zheng, J., J. Huang, Y. Mao, S. Liu and X. Sun et al., 2009. Immunoglobulin gene transcripts have distinct vhdjh recombination characteristics in human epithelial cancer cells. J. Biological Chem., 284: 13610-13619. DOI: 10.1074/jbc.M809524200

Zhou, X.X., W.H. Jia, G.P. Shen, H.D. Qin and X.J. Yu et al., 2006. Sequence variants in toll-like receptor 10 are associated with nasopharyngeal carcinoma risk. Cancer Epidemiol. Biomarkers Prev., 15: 862866. DOI: 10.1158/1055-9965.epi-05-0874

Zhu, X., C. Li, X. Sun, Y. Mao and G. Li et al., 2008. Immunoglobulin mrna and protein expression in human oral epithelial tumor cells. Applied Immunohistochem. Molecular Morphol., 16: 232238. DOI: 10.1097/PAI.0b013e31814c915a

Zhu, X., L. Wu, L. Zhang, P. Hao and S. Zhang et al., 2010. Distinct regulatory mechanism of immunoglobulin gene transcription in epithelial cancer cells. Cellular Molecular Immunol., 7: 279286. DOI: $10.1038 / \mathrm{cmi} .2010 .13$ 